\title{
Konzeptionen öffentlicher Dienstleistungen in Europa
}

Es scheint eine Epoche zu Ende zu gehen, in der der Bürger Anspruch auf eine stabile, preiswerte und qualitativ gute Versorgung mit öffentlichen Dienstleistungen hat, die der Staat als Eigenproduktion und in eigener Verantwortung zur Verfügung stellt. Seit Jahren wird liberalisiert, privatisiert und darüber diskutiert, was als angemessene Grundversorgung bezeichnet werden kann. Die Europäische Kommission treibt diese Entwicklung voran und versucht, ein gemeinschaftliches Regime öffentlicher Dienstleistungen aufzubauen. Es stellt sich die Frage, inwieweit dieses den Mitgliedstaaten noch ausreichend Freiheit belässt, um die öffentlichen Dienstleistungen weiterhin ihren gesellschaftlichen Traditionen und Bedürfnissen entsprechend gestalten zu können.

\section{Öffentliche Dienstleistungen und Daseinsvorsorge}

\subsection{BEGRIFFSBESTIMMUNG}

Unter öffentlichen Dienstleistungen werden solche Dienste verstanden, die von öffentlichen Händen im Interesse der Allgemeinheit gesichert und mit spezifischen Gemeinwohlverpflichtungen verknüpft werden. Wenn im Folgenden dieser Begriff verwendet wird, ist es also gleichgültig, wer die Dienste anbietet - eine Verwaltung, ein privates, öffentliches oder gemischtwirtschaftliches Unternehmen. Das „öffentlich" bezieht sich nicht auf die eigentumsrechtliche Konstruktion des Dienstleistungsunternehmens, sondern auf den Gemeinwohlcharakter der Dienste. Der Begriff dient hier als gemeinsame Klammer für recht unterschiedliche nationale und gemeinschaftliche Terminologien und Konzeptionen, die in diesem Zusammenhang verwendet werden. In Deutschland spricht man beispielsweise von „Daseinsvorsorge“.

Mit Daseinsvorsorge sind zum einen reale Dienstleistungen gemeint, zum anderen konzeptionelle Überlegungen. Der Begriff stammt zwar aus den 1930er Jahren, aber schon zuvor waren ähnliche Vorstellungen entwickelt worden. Sie blieben allerdings vage; ein differenzierteres Konzept der Daseinsvorsorge entstand nicht (Scheidemann 1991; Pielow 2001; Püttner 2002). Weder wurde ein ausgereifter gesellschaftswissenschaftlicher Ansatz entwickelt noch eine wirkliche rechtliche Dogmatik. Es ist daher Vorsicht geboten, wenn hier aus sozialwissenschaftlicher, nicht aus juristi- scher Perspektive dennoch versucht wird, die wichtigsten normativen Elemente einer aktuellen Konzeption der Daseinsvorsorge in Deutschland aufzulisten, von denen anzunehmen ist, dass sie weitgehend akzeptiert sind - allerdings nur von denjenigen, die dem Gedanken der Daseinsvorsorge grundsätzlich positiv gegenüberstehen.

\subsection{ELEMENTE DER DASEINS- VORSORGE}

(1) Bei der Daseinsvorsorge handelt es sich um „öffentliche“ Dienstleistungen, d. h. um Dienstleistungen, die dem Gemeinwohl dienen. Da es eine zeit- und raumlose, materiell-essenzialistische Definition des Gemeinwohls, des allgemeinen oder öffentlichen Interesses in freiheitlich-pluralistischen Demokratien nicht gibt, muss es immer wieder neu bestimmt werden. Im Zusammenhang mit Dienstleistungen der materiellen Infrastruktur bedeutet es zunächst: diskriminierungsfreier Zugang, Gleichbehandlung aller Bürger, Kontinuität und Qualität der Leistungen sowie Anpassungsfähigkeit. Der Begriff „Gemeinwohl“ kann auch soziale Preisgestaltung oder ein subventioniertes Leistungsangebot umfassen. Umweltschutz und Nachhaltigkeit sind ebenso der Daseinsvorsorge immanent wie beschäftigungspolitische oder wirtschaftsfördernde Aspekte. Daseinsvorsorge erfüllt eine anthropologische Basisfunktion; sie bedeutet mehr als nur eine minimale Grundversorgung.

(2) Die räumliche Perspektive spielt bei der Identifikation des Gemeinwohls eine wichtige Rolle. Je kleiner die Räume sind, auf die sich die öffentlichen Dienstleistungen beziehen, umso leichter können die Bedürfnisse der Menschen präzisiert werden. Da- seinsvorsorge und Subsidiarität hängen zusammen. Das Gemeinwohl soll möglichst bürgernah bestimmt werden.

(3) Bestimmte Leistungen können nicht ausschließlich durch die Gesetze des Marktes bereitgestellt werden. Eine klare Grenzziehung zwischen marktbezogenen und nicht-marktbezogenen oder wirtschaftlichen und nicht-wirtschaftlichen Dienstleistungen gibt es allerdings nicht. Ebenso wenig ist eine präzise Differenzierung zwischen einer erwerbsorientierten und einer gemeinwohlorientierten Leistungserstellung möglich.

(4) Auch wenn in der Vergangenheit bei öffentlichen Dienstleistungen oftmals monopolistische Strukturen herrschten, ist die Marktform des Monopols kein konstitutives Element der Daseinsvorsorge. Aus der Rechtsethik der Daseinsvorsorge sind öffentliche Monopole jedenfalls nicht begründbar. Daseinsvorsorgende Leistungen können grundsätzlich auch im Wettbewerb erbracht werden, je nach Versorgungssparte im Wettbewerb um den Markt oder im Wettbewerb auf dem Markt. Es herrscht allerdings ein gewisses Misstrauen gegen marktradikale Lösungen.

(5) Der Staat muss öffentliche Dienstleistungen nur gewährleisten. Er braucht sie

\footnotetext{
Gerold Ambrosius, Dr., ist Professor für Wirtschafts- und Sozialgeschichte an der Universität Siegen. Arbeitsschwerpunkte: Geschichte der europäischen Wirtschaftsintegration, der öffentlichen Dienstleistungssysteme und des institutionellen Wettbewerbs. e-mail:ambrosius@geschichte.uni-siegen.de
} 
nicht selbst zu produzieren und anzubieten. Daseinsvorsorge ist mit unterschiedlichen Eigentumsformen kompatibel. Die Leistungen, die nicht kostendeckend angeboten werden können, müssen vom Staat subventioniert werden.

(6) Öffentliche Dienstleistungen unterliegen einer intensiven Regulierung, die sich auf ganz unterschiedliche Tatbestände bezieht: auf die allgemeinen Marktbedingungen, die speziellen Bedingungen der Dienstleistungsmärkte, d. h. auf Missbrauchstatbestände, und die Rechtsformen von Unternehmen einerseits (Strukturregulierung) und auf die Unternehmensführung, den Umfang, die Qualität, die Preise der Dienste usw. andererseits (Verhaltensregulierung).

(7) Der Versuch, die Daseinsvorsorge von der allgemeinen Leistungsverwaltung dadurch abzugrenzen, dass nur solche Leistungen als daseinsvorsorgende bezeichnet werden, für die der Empfänger ein Entgelt zu zahlen hat - vielleicht sogar ein kostendeckendes -, macht keinen Sinn.

(8) Öffentliche Dienstleistungen können grundsätzlich von allen Gebietskörperschaften angeboten werden. Angesichts der großen Bedeutung der Gemeinden in diesem Zusammenhang werden aber in erster Linie diese mit der Daseinsvorsorge in Verbindung gebracht. Daseinsvorsorge und kommunale Selbstverwaltung sind auf das Engste verklammert. Daseinsvorsorgende Unternehmen stärken die unteren Gebietskörperschaften und somit den föderalen Staatsaufbau. Sie sind ein Stück materieller Demokratie.

(9) Mit Daseinsvorsorge werden in erster Linie die Dienste der netzgebundenen Infrastruktur (Wasser, Elektrizität, Gas, Information und Kommunikation, Verkehr) verbunden; es können aber auch die vielfältigen Dienste der sozialen und kulturellen Infrastruktur darunter subsumiert werden.

Diese Bausteine einer Konzeption der Daseinsvorsorge sind so vage formuliert, dass sie recht unterschiedliche Regime der Daseinsvorsorge zulassen. Damit bleibt die Frage unbeantwortet, ob die Privatisierungen und Entflechtungen öffentlicher Dienstleistungsunternehmen und die Liberalisierung öffentlicher Dienstleistungs- märkte in den letzten Jahren mit dem hier skizzierten Daseinsvorsorgekonzept noch vereinbar sind.

\section{Historische Wurzeln öffentlicher Dienstleis- tungen - das gemeinsame europäische Erbe}

Selbst wenn in manchen Ländern der Aufund Ausbau öffentlicher Dienstleistungen schneller erfolgte als in anderen, ändert das nichts an der Tatsache, dass der Staat am Ende des 19. Jahrhunderts überall in Europa bestimmte Dienstleistungen in eigene Regie übernahm oder ihr Angebot zumindest gewährleistete und nach Gemeinwohlkriterien regulierte. Öffentliche Dienstleistungen wurden Bestandteil eines - wie es die Europäische Kommission heute ausdrückt - „europäischen Gesellschaftsmodells". Dabei hatte das öffentliche Engagement ganz unterschiedliche Ursachen: Ein liberaler Antimonopolismus wandte sich gegen die Gefahr der privaten Ausnutzung infrastruktureller bzw. natürlicher Monopole. Ein sozialkonservativer Paternalismus wollte den Missständen des kapitalistischen Systems mit öffentlicher Sozialreform begegnen, zu der auch öffentliche Leistungen gehörten. Ein sozialistischer Reformismus versuchte, antikapitalistische Ordnungsprinzipien im bestehenden System zu verankern. Ein republikanischer Interventionismus zielte darauf ab, gesellschaftliche Interessen als öffentliche zu definieren und den Primat der Politik gegenüber der Ökonomie zumindest in diesem Bereich durchzusetzen. Nicht alle Strömungen oder Paradigmen waren in den europäischen Staaten gleich stark vertreten. Fast überall gab es aber eine Gemengelage dieser unterschiedlichen Motive.

Mit öffentlichen Dienstleistungen war schon im ausgehenden 19. Jahrhundert der Gedanke des gesellschaftlichen Ausgleichs verbunden. Bestimmte Güter und Dienste sollten aus Gründen des Gemeinwohls nicht oder nicht ausschließlich durch die Gesetze des Marktes bereitgestellt, sondern entweder hoheitlich reguliert oder in öffentlichen Unternehmen produziert werden. Die meisten Staaten Europas entschieden sich für öffentliche Eigenproduktion, allerdings weniger aus ideologischen, sondern mehr aus pragmatischen Gründen:
Öffentliche Unternehmen ließen sich einfach leichter im Interesse der Allgemeinheit steuern. Hierarchische Lenkung war effektiver als vertragliche. Es entwickelten sich Dienstleistungsregime, die hinsichtlich der Marktformen, des Verhältnisses von öffentlichen und privaten Unternehmen oder der Regulierungsintensität durchaus nicht identisch waren. Die Faktizität öffentlicher Dienstleistungen als Aufgabenzuwachs der selbst produzierenden oder gewährleistenden Leistungsverwaltung manifestierte sich aber fast überall in Europa ähnlich.

Somit war die Geschichte Europas durch die Suche nach einem gesellschaftlichen Entwicklungsmodell gekennzeichnet, das jenseits von extremem Liberalismus und revolutionärem Sozialismus privatwirtschaftliche Effizienz und gemeinwirtschaftliche Solidarität miteinander verbinden sollte. Schon im 19. Jahrhundert dienten öffentliche Dienstleistungen der wirtschaftlichen und sozialen, letztlich der gesellschaftlichen Kohäsion. Seit dieser Zeit war die öffentliche Steuerung von Dienstleistungsunternehmen vornehmlich durch Verhaltens- oder Aufgabenregulierung gekennzeichnet. Sie legte den Unternehmen beim Ausbau der Netze, bei der Qualität der Dienste und beim Preis der Leistungen Gemeinwohlverpflichtungen auf. Damit unterschied sich Europa von Ländern wie den USA, bei denen die Steuerung zumindest tendenziell auf Struktur- oder Wettbewerbsregulierung ausgerichtet war.

\section{Konzeptionen öffentlicher Dienstleistungen in Europa}

Im Hinblick auf die Bedeutung öffentlicher Dienstleistungen im Recht kann zwischen zwei Staatengruppen unterschieden werden: einerseits Staaten, in denen der Begriff eine erhebliche rechtliche, auch verfassungsrechtliche Bedeutung hat, wie in Frankreich und Staaten mit romanischer oder Frankreich nahestehender Rechtstradition - Italien, Spanien, Portugal, Belgien oder Griechenland (Typ 1) -, andererseits Staaten, in denen der Begriff existiert, ohne aber rechtlich aufgeladen zu sein. Zu Letzterem zählen Großbritannien, Deutschland, Niederlande, Irland oder die nordischen Länder (Typ 2) (CEEP 1996). Allerdings muss zwischen rechtlicher Doktrin und gesell- 
schaftspolitischer Konzeption unterschieden werden, wobei sich Letztere, wie gesagt, in den europäischen Staaten ebenfalls recht unterschiedlich ausprägte. Wenn einige dieser Konzeptionen im Folgenden kurz dargelegt werden, ist dies nur durch radikale Vereinfachungen möglich - Vereinfachungen im Hinblick auf die Konzepte als solche, die unterschiedlichen gesellschaftspolitischen Strömungen innerhalb der angesprochenen Länder und die Unterschiede zwischen praktischer Politik und konzeptionellen Überlegungen. Manche Veränderungen der letzten Jahre sind mehr der finanzpolitischen Not geschuldet als der politischen Überzeugung (Lippert 2005; CEEP 1995, 1996; Lyon-Caen/ChampeilDesplats 2001; Hemmer/Hollos 2003; Höferl 2004; Hollos 2003).

\subsection{TYP 1}

\section{FRANKREICH}

Frankreich ist das Land, das mit dem Begriff des „service public“ das dezidierteste Konzept öffentlicher Dienstleistungen entwickelt hat (Pielow 2001; Pielow 2002; Bullinger 2003; Le Masne 2007). Neben seiner Relevanz als Doktrin des Verwaltungsrechts besitzt der Begriff eine erhebliche gesellschafts- und staatspolitische bzw. -philosophische Bedeutung. Traditionell sind öffentliche Dienstleistungen ein Element republikanisch-demokratischer Ordnung. Der service public ist nicht auf das beschränkt, was man in Deutschland als daseinsvorsorgende Leistungen im engeren Sinne bezeichnet, also vornehmlich netzgebundene Infrastrukturen, sondern kann neben Hoheits- bzw. Souveränitätsaufgaben und Dienstleistungen des Sozialstaates ebenso unternehmerisch-wirtschaftliche Aktivitäten der Gebietskörperschaften umfassen. Öffentliche Dienstleistungen können also marktbestimmt und nicht marktbestimmt sein. Diesem weiten Bereich der services publics entsprechen ganz unterschiedliche Eigentums- und Organisationsformen. Selbst wenn öffentliche Unternehmen eine wichtige Rolle spielen, herrscht doch traditionell ein eigentumsrechtlicher Pluralismus. Allerdings wird die Angebotserstellung öffentlicher Dienstleistungen an Private nur delegiert und nicht übertragen. Ebenso gibt es vielfältige Marktformen; der service public kann sowohl im Monopol als auch im Wettbewerb erbracht werden. Eine radikale Wettbe- werbsdoktrin wird allerdings strikt abgelehnt. Mehr oder weniger abhängige Regulierungsagenturen sorgen für eine gemeinwohlorientierte Steuerung des Verhaltens und der Gewinne der dienstleistenden Unternehmen. Insgesamt besitzt der service public eine herausragende Bedeutung in der Verantwortung der „Republik“ ihren Bürgern gegenüber.

\section{ITALIEN UND SPANIEN}

In Italien und Spanien hat der „servizio pubblico“bzw. der „servicio público“ zwar nie den gleichen Stellenwert gehabt wie in Frankreich der service public, im Vergleich $\mathrm{zu}$ anderen Ländern ist er aber ebenfalls von relativ großer Bedeutung - als rechtliche Doktrin ebenso wie als gesellschaftspolitisches Konzept. Beide Länder sind durch das französische Paradigma beeinflusst worden. Besonders in Italien sind öffentliche Dienstleistungen lange Zeit mit öffentlichen Eigentums- und monopolistischen Marktformen identifiziert worden, wobei allerdings seit einigen Jahren - wie in anderen Ländern auch - ein Umdenken stattfindet, d.h. privatisiert und liberalisiert wird (Ferrari 2001; Cassese 1995; Essebier 2005). Mehr oder weniger unabhängige Regulierungsorgane sollen auch hier dafür sorgen, dass die Gemeinwohlverpflichtungen von den privatisierten, im Wettbewerb stehenden Dienstleistungsunternehmen eingehalten werden. In Spanien findet ein ähnlicher paradigmatischer Wandel statt, wobei private Unternehmen bei der öffentlichen Aufgabenerfüllung - über Konzessionen gesteuert - traditionell eine größere Rolle spielen (Gonzales-Varas 2002; des Guago Castiella/Pielow 1995; MaleretGarcia 2001; Jimenez-Blanco 1995). Wie in Frankreich ist in beiden Ländern die Vorstellung vom Grundrecht der Bürger auf eine angemessene Versorgung mit öffentlichen Dienstleistungen trotz Liberalisierung und Privatisierung noch immer präsent, sodass die Strukturregulierung zur Durchsetzung wettbewerblicher Marktformen durch eine starke Verhaltensregulierung zur Durchsetzung bürgerorientierter Gemeinwohlverpflichtungen ergänzt wird.

\subsection{TYP 2}

\section{GROßBRITANNIEN}

Großbritannien ist sicherlich der Mitgliedstaat der Europäischen Union (EU), in dem die Vorstellung von öffentlichen Dienstleistungen als wichtiger Ausdruck des gesellschaftlichen Zusammenhalts am schwächsten entwickelt ist (Sturm/Müller 2002; Lippert 2005; Essebier 2005; Schneider 2006). Mangels eines Verwaltungsrechts bzw. einer Verwaltungsgerichtsbarkeit ist eine dem service public oder auch der Daseinsvorsorge vergleichbare Konzeption nicht entwickelt worden. Mit den „public utilities" sind zwar ebenfalls soziale Aufgaben oder Gemeinwohlverpflichtungen verbunden. Auch in Großbritannien soll eine Grundversorgung mit lebensnotwendigen Diensten bzw. Gütern gesichert werden. Es gibt aber ebenso die liberale Tradition, die dazu führte, dass Großbritannien das erste Land war, das seit den 1980er Jahren öffentliche Dienstleistungsunternehmen konsequent privatisierte und öffentliche Dienstleistungsmärkte liberalisierte, wobei die Marktöffnung eher eine untergeordnete Rolle spielte. Der Glaube an das private Eigentum ist das eigentliche Kennzeichen des britischen Modells. Grundsätzlich soll die Infrastruktur in öffentlichen Händen bleiben, während die Produktion und Lieferung der Dienstleistungen durch Private erbracht wird. Bereits privatisierte Netze werden in jüngster Zeit sogar wieder verstaatlicht. Der Staat soll sich auf die Aufsicht und die wettbewerbsfördernde Regulierung des Marktes beschränken. Die privaten Unternehmen werden durch sektorale Regulierungsbehörden überwacht, die mit Anreizregulierung versuchen, Wettbewerbsbedingungen $\mathrm{zu}$ simulieren, um die angestrebten Produktivitäts- und Qualitätsverbesserungen zu erreichen. Diese marktregulierende Aufgabe soll möglichst dezentral und marktnah ausgeübt werden. Mit der konsequenten Privatisierung und den starken und unabhängigen Regulierungsorganen ist Großbritannien stärker dem liberal-marktwirtschaftlichen Paradigma verpflichtet als die meisten anderen europäischen Staaten.

\section{SCHWEDEN}

Schweden kennt zwar ebenfalls keine dem service public vergleichbare juristische Konstruktion und gesellschaftspolitische Konzeption (Lippert 2005; Schneider 2006). Der öffentliche Sektor und damit die öffentlichen Dienstleistungen spielen aber im Rahmen des Wohlfahrtsstaates eine wichtige Rolle. In Schweden ist man nicht wie in Großbritannien den Weg der mate- 
riellen Privatisierung gegangen, sondern hat versucht, durch Liberalisierung die Märkte dem Wettbewerb zu öffnen. Seit Mitte der 1990er Jahren herrscht fast in allen Sektoren Konkurrenz. Anders als in Großbritannien übt der Staat weiterhin über öffentliche Unternehmen Einfluss aus, die aber zunehmend im Wettbewerb stehen und stärker marktorientiert agieren. Die Regulierungsbehörden sind relativ abhängig, dienen vor allem der Marktbeobachtung und arbeiten nur sporadisch mit Wettbewerbsinstrumenten wie Benchmarking oder Price Caps. Letztlich haben die strukturellen Veränderungen der öffentlichen Dienstleistungen in jüngster Zeit deren konzeptionelle Verankerung im schwedischen Modell des zentralen korporativ organisierten Wohlfahrtsstaates mit starken Steuerungsmöglichkeiten kaum berührt.

Insgesamt haben alle Mitgliedstaaten der EU in den letzten Jahren zwar insofern einen paradigmatischen Wandel vollzogen, als das private Eigentum, der wettbewerbliche Markt und die unabhängige Regulierungsagentur eine größere Bedeutung bekommen haben. Da aber noch genügend nationalspezifische Eigenarten geblieben sind, ist durchaus nicht klar, ob sich mit diesem Wandel die unterschiedlichen Regime - die theoretischen Konzepte wie die realen Strukturen - wie oft behauptet angeglichen haben.

\section{Gemeinschaftliches Konzept öffentlicher Dienstleistungen}

Angesichts dieser unterschiedlichen Dienstleistungskonzepte stellt sich die Frage, ob es so etwas wie eine gemeinschaftliche Konzeption gibt. Bis 2001 verwendete die Kommission in ihren einschlägigen Mitteilungen den jeweiligen nationalspezifischen Begriff; in der deutschen Übersetzung wurde der der Daseinsvorsorge benutzt. Seit 2003 ist sie - abgesehen von dem im ursprünglichen Vertrag der Europäischen Wirtschaftsgemeinschaft (EWG) benutzten Begriff der „Dienstleistungen von allgemeinem wirtschaftlichem Interesse " - mit den „Dienstleistungen von allgemeinem Interesse“ zu einer neuen Terminologie übergegangen und signalisiert damit stärker als bis dahin, dass sie eine eigenständig ge- meinschaftliche Konzeption aufzeigen will. Das Projekt einer gemeinschaftlichen Rahmenrichtlinie für Dienstleistungen von allgemeinem Interesse wird von ihr verfolgt.

Um einer gewissen Systematik zu folgen, wird im Folgenden zwischen der bürgerrechtlichen, der wirtschaftsordnungspolitischen und der gemeinwohlbezogenen Perspektive unterschieden (Becker 2005).

\subsection{BÜRGERRECHTLICHE PERSPEKTIVE}

In den einschlägigen Mitteilungen der Kommission und den Veröffentlichungen des Parlaments seit Mitte der 1990er Jahre wird die diskriminierungsfreie, langfristig gesicherte, preiswerte Grundversorgung mit bestimmten Dienstleistungen als „gemeinsamer Wert" der europäischen Gesellschaften, als „Grundrecht“ der Europäer oder als „Pfeiler einer europäischen Staatsbürgerschaft“, als „Kern des europäischen Gesellschaftsmodells" bezeichnet (Ambrosius 2006). 1996 wurde Art. 16 in den EGVertrag aufgenommen, durch den die Gemeinschaft und die Mitgliedstaaten aufgefordert werden, dafür zu sorgen, dass „die Grundsätze und Bedingungen für das Funktionieren der Dienste so gestaltet sind, dass sie ihren Aufgaben nachkommen können". 2000 wurden die öffentlichen Dienstleistungen im Art. 36 der „Charta der Grundrechte der Europäischen Union" erwähnt. Schließlich sind sie durch das „Protokoll über Dienste von allgemeinem Interesse“ Bestandteil des Reformvertrags von 2007. Dort ist ebenfalls von ,gemeinsamen Werten der Union in Bezug auf Dienste von allgemeinem wirtschaftlichem Interesse" die Rede.

Lässt man das Pathos und die strategischen bzw. taktischen Ziele dieser Texte außer Betracht, ist zumindest eine veränderte Perspektive der europäischen Institutionen gegenüber der Zeit vor Maastricht zu konstatieren. Öffentliche Dienstleistungen sind im europäischen Kontext sicherlich aufgewertet worden. Über die Bedeutung dieser veränderten Perspektive, insbesondere über die des Art. 16, gibt es natürlich unterschiedliche Auffassungen. Unmittelbar rechtliche Konsequenzen hat er wohl nicht. Er begründet jedenfalls kein subjektives Recht auf ein konkretes Versorgungsniveau. $\mathrm{Ob}$ aus Art. 16 allerdings nur der politische Wunsch abgeleitet werden kann, beim Übergang zu einer konsequent wettbewerblichen Ordnung Anpassungs- schwierigkeiten $\mathrm{zu}$ vermeiden, erscheint fraglich. Immerhin beinhaltet er einen Gestaltungsauftrag.

\subsection{WIRTSCHAFTSORDNUNGS- POLITISCHE PERSPEKTIVE}

Kommission und Parlament sind der Meinung, dass sich aus dem Europarecht kein prinzipieller Widerspruch zwischen der liberalen Marktverfassung und den Dienstleistungsunternehmen mit besonderen oder ausschließlichen Rechten ableiten lässt (Nettesheim 2002; Becker 2005). Es gilt erstens der Grundsatz der Gestaltungsfreiheit. Den Mitgliedstaaten steht es danach frei, das Gemeinwohl zu definieren und gemeinwohlorientierte Dienste selbst bereitzustellen oder zu gewährleisten. Sie brauchen sich also nicht im Sinne einer Strukturregulierung darauf zu beschränken, die Ordnung für wettbewerbliche Dienstleistungsmärkte zu schaffen, sondern können im Sinne einer Verhaltensregulierung die Gemeinwohlziele definieren und realisieren, die im privaten Markt nicht eingelöst werden. Allerdings muss zweitens der Grundsatz der Verhältnismäßigkeit gewahrt werden. Das bedeutet, dass es sich bei den Diensten, mit deren Bereitstellung nach Art. 86 EG-Vertrag ein Unternehmen ,betraut' wird, tatsächlich um „öffentliche“ handeln muss. Die Maßnahmen, die zur Verwirklichung von Gemeinwohlverpflichtungen ergriffen werden, dürfen den innergemeinschaftlichen Handel nicht mehr beeinträchtigen, als dies zur Verwirklichung der gewünschten Ziele unbedingt notwendig ist. Drittens ist der Grundsatz der Neutralität hinsichtlich der eigentumsrechtlichen Form öffentlicher Dienstleistungsunternehmen von Anfang an im EG-Vertrag verankert gewesen.

Diese pauschale Einschätzung wäre auf vielfältige Weise zu differenzieren, u. a. im Hinblick auf die europäischen Organe. Im Europäische Parlament dominiert wohl die Auffassung, dass - wie es in einer Entschließung von 2001 heißt - „eine Weiterentwicklung der Daseinsvorsorge auf der Grundlage des Modells der Sozialen Marktwirtschaft ein Kernelement der europäischen Wirtschafts-, Finanz- und Sozialpolitik sein muss ..." (Europäisches Parlament 2001, Ziffer L). Gemeinwohlorientierte Dienstleistungen werden als konstitutive Elemente einer europäischen Wirtschafts- und Sozialordnung propagiert, die in keiner Weise im Widerspruch 
zur Wettbewerbs- bzw. Binnenmarktordnung stehen. Auch wenn das Parlament immer wieder das Prinzip der Verhältnismäßigkeit hervorhebt und die Bereitstellung im Wettbewerb grundsätzlich positiv beurteilt, steht es einer radikalen Öffnung der Dienstleistungsmärkte doch skeptisch gegenüber. Die Liberalisierung der Wasserversorgung wird beispielsweise abgelehnt. Das Parlament ist bereit, den öffentlichen Dienstleistungen hinsichtlich der Wahrnehmung der Freiheitsrechte der EU-Bürger und des sozialen und territorialen Zusammenhalts der Gemeinschaft tatsächlich besondere Bedeutung beizumessen. Seine Auffassung dürfte am ehesten der hier skizzierten Version eines deutschen Daseinsvorsorge-Konzepts entsprechen.

Was die Kommission betrifft, so wird es ebenfalls nur schwer gelingen, sie auf eine wirklich eindeutige Position festzulegen. Einerseits deuten manche Stellungnahmen auf ein marktkonsequentes Wettbewerbskonzept hin, nach dem alle öffentlichen Dienstleistungen ausgeschrieben und Beihilfen immer angemeldet werden müssen. Nach dieser Auffassung schließen sich Gemeinwohl und Konkurrenz gerade nicht aus, weil der Wettbewerb für höhere Effizienz und Wirtschaftlichkeit bei der Versorgung mit öffentlichen Diensten sorgt. Es gilt der Primat des Wettbewerbs unter gleichen Bedingungen. Andererseits geht die Kommission sehr gelassen mit der durch den EG-Vertrag angedeuteten Wirtschaftsverfassung und ihren Wettbewerbs- bzw. Binnenmarktregeln um. Dienstleistungsunternehmen mit besonderen oder ausschließlichen Rechten sollen dort aktiv werden, wo der Markt entsprechende Dienste nicht ausreichend bereitstellt. Einen Widerspruch zwischen Binnenmarkt und Gemeinwohl sieht sie aus dieser Perspektive jedenfalls nicht. Insgesamt konzentriert sich die Kommission seit geraumer Zeit fast ausschließlich auf wettbewerbsrechtliche Fragen, zurzeit insbesondere auf die Ausweitung des Ausschreibungswettbewerbs. Ihrer Meinung nach sollen die Staaten die Produktion und Bereitstellung öffentlicher Dienstleistungen weitestgehend den wettbewerblichen Märkten überlassen, die Netze durch Wettbewerbssurrogate stärker dem Konkurrenzdruck aussetzen und sich auf die Rolle des Gewährleisters und Regulators beschränken. Bei ihren Vorschlägen zur Steuerung von Dienstleistungsunternehmen steht daher auch die Struktur- regulierung im Vordergrund. Sie hat außerdem eine eindeutige Affinität für die Erbringung der Dienste durch Private. Der Ansatz der Kommission entspricht somit nicht oder nur bedingt dem hier skizzierten Konzept der Daseinsvorsorge.

Der Gerichtshof beschäftigt sich in diesem Zusammenhang mit verschiedenen Themenbereichen. Er trifft Entscheidungen hinsichtlich der Klassifikation von wirtschaftlichen und nicht-wirtschaftlichen Diensten; nur Erstere werden ja vom europäischen Wettbewerbsregime überhaupt erfasst. Er grenzt Leistungen wie nationale Bildungssysteme oder öffentlichrechtliche Sozialversicherungen aus dem Einflussbereich des EG-Vertrages aus. Allerdings finden seiner Meinung nach die Binnenmarktvorschriften des Vertrages auf Dienstleistungen nicht-wirtschaftlicher Art dann Anwendung, wenn sich die Gebietskörperschaften Dritter bedienen, um diese Dienste anzubieten. Weiter hat das Gericht wichtige Urteile u. a. zum Problem der Beihilfen und der Ausschreibungen gefällt. Diesen Urteilen liegt eine markt- bzw. wettbewerbskonsequente Doktrin zugrunde, ohne allerdings die besonderen Probleme bei der Bereitstellung oder Gewährleistung öffentlicher Dienstleistungen völlig außer Acht zu lassen. Ohne Zweifel wird aber das Europarecht primär im Sinne des Wettbewerbsparadigmas interpretiert. Aus Sicht des deutschen Konzepts der Daseinsvorsorge ist das eine sehr einseitige Interpretation.

\subsection{GEMEINWOHLBEZOGENE PERSPEKTIVE}

Kommission und Parlament halten es weder für möglich noch für erstrebenswert, eine „einheitliche, umfassende europäische Definition" des Gemeinwohls von öffentlichen Dienstleistungen zu entwickeln (Ambrosius 2006). Sie wiederholen gebetsmühlenartig die Notwendigkeit, die unterschiedlichen kulturellen Traditionen oder Identitäten zu respektieren. Es gibt kein Gemeinschaftsrecht, das die Gemeinwohlverpflichtungen öffentlicher Dienstleistungen festlegt. Dennoch schreitet vor allem die Kommission auf dem Weg zur Harmonisierung auch in dieser Hinsicht zügig voran. Für die Dienstleistungen von allgemeinem Interesse sind von ihr im Weißbuch von 2004 folgende neun „Leitprinzipien" formuliert worden:
- Voraussetzungen für bürgernahe öffentliche Regulierung schaffen,

- die Ziele öffentlicher Dienste in wettbewerbsfähigen, offenen Märkten erreichen,

- Kohäsion und universellen Zugang sicherstellen,

- ein hohes Qualitäts-, Versorgungssicherheits- und Schutzniveau aufrechterhalten, - die Rechte der Verbraucher und Nutzer sichern,

- Monitoring und Leistungsevaluierung durchführen,

- die Verschiedenheit von Dienstleistungen und Situationen berücksichtigen,

- mehr Transparenz schaffen,

- Rechtssicherheit gewährleisten.

Diese Leitprinzipien werden zwar ausgeführt, bleiben aber vage. Konkreter formuliert sind die Kriterien, die sich auf die „Dienstleistungen von allgemeinem wirtschaftlichem Interesse" beziehen: Kontinuität, Qualität, Erschwinglichkeit, Nutzerund Verbraucherschutz, freier Netzzugang und Interkonnektivität netzgebundener Dienste. Diese Kriterien fließen in die Konzepte der „Verpflichtungen des öffentlichen Dienstes" (Verkehrs- und Energierichtlinien) und des „Universaldienstes“ (Kommunikations- und Postrichtlinien) ein, die im Zusammenhang mit den sektoralen Liberalisierungsrichtlinien für netzgebundene Dienste entwickelt wurden. Ein Universaldienst im gemeinschaftsrechtlichen Sinne meint die Gewährleistung eines bestimmten Angebots an öffentlichen Diensten. Über die Art und Weise, wie dies geschehen soll, werden keine Aussagen gemacht. Die Mitgliedstaaten brauchen also auch nicht bestimmte Unternehmen mit der Bereitstellung von öffentlichen Diensten zu „betrauen“. Insofern sind Universaldienste und Dienstleistungen von allgemeinem (wirtschaftlichem) Interesse nicht identisch, vielmehr sind Erstere Letzteren ein- oder untergeordnet (Linder 2004). Das Parlament fordert in diesem Zusammenhang eine stärkere Berücksichtigung sozialer Belange.

Wie sehr die Konkretisierung des daseinsvorsorgenden Gemeinwohls auf Gemeinschaftsebene die Entwicklung der öffentlichen Dienstleistungen in den Mitgliedstaaten beeinflussen kann, macht die „Richtlinie über den Universaldienst und Nutzerrechte bei elektronischen Kommunikationsnetzen und -diensten " von 2002 deutlich. Für andere netzgebundene Dienstleistungen gibt es solche präzisen Vorgaben 
allerdings noch nicht. Lässt man die zahlreichen Veröffentlichungen der europäischen Organe der letzten Jahre, insbesondere die der Kommission, Revue passieren, so besteht ein Ungleichgewicht zwischen den sektoralen Vorschriften zur Liberalisierung und den übergreifenden Gemeinwohlverpflichtungen sowie deren sektorspezifischer Konkretisierung. Es ist nicht geklärt, wie sich Einheit und Vielfalt in der Union bzw. Harmonisierung und Subsidiarität in diesem Zusammenhang verhalten sollen. Ob die verschiedenen Hinweise auf das Subsidiaritätsprinzip und der Bezug auf die regionale und lokale Selbstverwaltung im Reformvertrag sowie das „Protokoll über Dienste von allgemeinem Interesse" von Bedeutung sind, wird sich erst noch zeigen. Der Wille der Kommission, den Rahmen für die nationalen Dienstleistungsregime enger zu fassen und präziser auszugestalten, ist unverkennbar. Die Diskussion um eine gemeinschaftliche Rahmenrichtlinie für Dienstleistungen von allgemeinem Interesse bleibt aktuell. Offen ist weiterhin, ob es sich beim Universaldienst nur um eine minimale Grundversorgung oder um mehr handelt. Auf den ersten Blick erscheinen manche Veröffentlichungen der europäischen Institutionen als Umsetzung der hier vorgenommenen Interpretation des Daseinsvorsorge-Konzepts in Deutschland. Papier ist aber bekanntlich geduldig. Die Politik der Kommission und die Urteile des Gerichtshofes sprechen eine andere Sprache.

\section{Europäisierung nationaler Dienstleistungssysteme}

Die Kommission belässt es nicht bei dem Versuch, eine gemeinschaftliche Dienstleistungskonzeption zu entwickeln. Sie versucht auch, die Organisation der nationalen Dienstleistungsregime direkt zu beeinflussen. Hauptsächlich vollzieht sich dies über die von der Kommission geforderte oder empfohlene gemeinschaftseinheitliche Organisation der Regulierung, Finanzierung und Evaluierung öffentlicher Dienstleistungen.

Auf ihr Drängen werden unabhängige Regulierungsstellen bzw. -agenturen eingerichtet, die in Europa bisher eher selten waren. Einige Mitgliedstaaten haben solche Stellen sogar in Bereichen geschaffen, für die es noch keine gemeinschaftlichen Vorschriften gibt. Es wird eine enge $\mathrm{Zu}-$ sammenarbeit der nationalen Regulierungsorgane empfohlen. Im Eisenbahnund Kommunikationssektor enthalten die Rechtsvorschriften der Gemeinschaft eine Klausel, nach der die nationalen Agenturen ausdrücklich verpflichtet sind, ihre Entscheidungsprinzipien abzustimmen. Außerdem sind europäische Gremien entstanden, die für die Kohärenz der Regulierungspolitiken sorgen sollen: der Rat der europäischen Energieregulatoren (CEEP), der Europäische Ausschuss für die Regulierung der Post (CERP) oder die Joint Aviation Authorities (JAAs).

Bei der Finanzierung der Ausgleichszahlungen für öffentliche Dienstleistungen, die nicht rentabel am Markt erbracht werden können, steht es den Mitgliedstaaten grundsätzlich frei, die Art zu wählen, die sie für die günstigste halten: finanzielle Direkthilfe aus dem Staatshaushalt, besondere oder ausschließliche Rechte, Beiträge von Marktteilnehmern, Gebührenvereinheitlichung und Finanzierung nach dem Solidarprinzip. Es gibt allerdings sektorspezifische Bestimmungen zur Berechnung der Ausgleichszahlungen. Um für mehr Rechtssicherheit und Transparenz zu sorgen, hat die Kommission außerdem einen „Gemeinschaftsrahmen für staatliche Beihilfe, die als Ausgleich für die Erbringung öffentlicher Dienstleistungen gewährt werden", geschaffen.

Schließlich soll die Evaluierung der öffentlichen Dienstleistungssysteme harmonisiert werden, um die soziale und territoriale Kohäsion der Gemeinschaft und den grenzüberschreitenden Austausch empfehlenswerter Praktiken bei der Bereitstellung öffentlicher Dienste zu fördern. Die Kommission hat ein Konzept der Evaluierung erstellt, das auf drei Bewertungsansätzen beruht: sektorale vertikale Bewertung, sektorübergreifende horizontale Bewertung und regelmäßige Erhebungen zur Verbraucherzufriedenheit. Die Überprüfung erfolgt anhand sozialer, wirtschaftlicher und technischer Kriterien, die möglichst standardisiert werden sollen. Insgesamt üben diese Vorgaben und Empfehlungen einen erheblichen Angleichungsdruck auf die nationalen Dienstleistungskonzepte und -politiken aus.

\section{Fazit}

Privatisierung und Liberalisierung haben in den letzten Jahren zu tief greifenden Veränderungen in den öffentlichen Dienstleistungsregimen Europas geführt. Den Hintergrund dieser realen Veränderungen bildet ein paradigmatischer Wandel, der intensiveren Wettbewerb, mehr Privateigentum und stärkere Regulierung in allen Dienstleistungsbereichen fordert. Das konzeptionelle Umdenken findet in allen Staaten der EU statt, allerdings in nationalspezifischen Ausprägungen. Die traditionellen Dienstleistungskonzepte prägen auch die aktuellen, sodass weiterhin deutliche Unterschiede bestehen. Die Europäische Kommission, die die Liberalisierung maßgeblich vorangetrieben hat, bemüht sich darum, ein gemeinschaftliches Konzept öffentlicher Dienstleistungen zu entwickeln, das einerseits den Mitgliedstaaten ausreichende Freiräume zur nationalen Ausgestaltung lassen soll, das andererseits aber mehr sein will als nur ein unverbindlicher Rahmen. Erste Konturen eines solchen Konzepts sind bereits erkennbar. Wie stark der dadurch erzeugte Harmonisierungsdruck sein wird und wie sehr es zur konzeptionellen und damit zur realen Konvergenz führt, ist noch nicht abzusehen. 
Ambrosius, G. (2006): Öffentliche Dienstleistungen und Gemeinwohl in der europäischen Vorsorgepolitik, in: Ambrosius, G./Schmitt-Egner, P. (Hrsg.): Europäisches Gemeinwohl - Historische Dimension und aktuelle Bedeutung, Baden-Baden, S. 183-204

Ambrosius, G. (2007): Die Europäisierung des Gemeinwohls und die Entwicklung der öffentlichen Dienstleistungen in Europa, in: Bräunig, D./ Greiling, D. (Hrsg.): Stand und Perspektiven der Öffentlichen Betriebswirtschaftslehre II, Berlin, S. 99-110

Becker, P. (2005): Europäische Daseinsvorsorge. Die Politik der EU zwischen Wettbewerb und Gemeinwohlverpflichtung, Berlin

Bullinger, M. (2003): Französischer Service Public und deutsche Daseinsvorsorge, in: Juristenzeitung 12, S. 597-604

Cassese, S. (1995): Les services publics an Italie, in: CEEP (Hrsg.): Europe, concurrence et service public, Paris, S. 109ff.

Rat der Europäischen Energieregulatoren (CEEP) (Hrsg.) (1995): Europe, concurrence et service public, Paris

Rat der Europäischen Energieregulatoren (CEEP) (Hrsg.) (1996): Europa, Wettbewerb und öffentliche Dienstleistungen, Berlin

Des Guago Castiella, I./Pielow, J.-C. (1995): Das spanische Konzept des Servicio Publico, in: Recht der Energiewirtschaft 6, S. 225-233

Essebier, J. (2005): Dienstleistungen von allgemeinem wirtschaftlichem Interesse und Wettbewerb. Eine rechtsvergleichende Untersuchung zu Art. 86 II EG, Baden-Baden

Europäisches Parlament (2001): Entschließung zu der Mitteilung der Kommission "Leistungen der Daseinsvorsorge in Europa" vom 13.11., Brüssel

Ferrari, E. (2001): Service public et activites d'untilié publique en Italie, in: Lyon-Caen, A./Champeil-Desplats, V. (Hrsg.): Services publics et droits fondamentaux dans la construction européenne, Paris, S. 66ff.

Gonzales-Varas, S. (2002): Die Auffassung der "öffentlichen Dienstleistungen" oder Daseinsvorsorge im spanischen Recht (servicios públicos), in: Hrbek, R./Nettesheim, M. (Hrsg.): Europäische Union und mitgliedstaatliche Daseinsvorsorge, Baden-Baden, S. 210-222

Hemmer, D./Hollos, B. N. (2003): Privatisierung und Liberalisierung öffentlicher Dienstleistungen in der EU-15, Eisenbahn und ÖPNV, Wien Höferl, A. (2004): Privatisierung und Liberalisierung öffentlicher Dienstleistungen in der EU-15, Telekommunikation, Wien

Hollos, B. M. (2003): Privatisierung und Liberalisierung öffentlicher Dienstleistungen in der EU-15, Wasser und Abwasser, Wien
Jimenez-Blanco, A. (1995): Les services publics en Espagne, in: CEEP (Hrsg.): Europe, concurrence et service public, Paris, S. 163ff.

Le Masne, P. (2007): Les services publics. Approches économiques et enjeux sociaux, Paris

Linder, C. (2004): Daseinsvorsorge in der Verfassungsordnung der Europäischen Union. Primärrechtliche Grundsätze eines Rechts der Dienste von allgemeinem wirtschaftlichem Interesse, Frankfurt am Main u.a. Lippert, I. (2005): Öffentliche Dienstleistungen unter EU-Einfluss. Liberalisierung - Privatisierung - Restrukturierung - Regulierung, Berlin Lyon-Caen, A./Champeil-Desplats, V. (Hrsg.) (2001): Services publics et droits fondamentaux dans la construction européenne, Paris Maleret-Garcia, E. (2001): Les services public en espagne, in: Lyon-Caen, A./Champeil-Desplats, V. (Hrsg.): Services publics et droits fondamentaux dans la construction européenne, Paris, S. $32 \mathrm{ff}$.

Nettesheim, M. (2002): Mitgliedstaatliche Daseinsvorsorge im Spannungsfeld zwischen Wettbewerbskonformität und Gemeinwohlverantwortung, in: Hrbek, R./Nettesheim, M. (Hrsg.): Europäisches Union und mitgliedstaatliche Daseinsvorsorge, Baden-Baden, S. 29-64

Pielow, J.-C. (2001): Grundstrukturen öffentlicher Versorgung. Vorgaben des Europäischen Gemeinschaftsrechts sowie des französischen und des deutschen Rechtes unter besonderer Berücksichtigung der Elektrizitätswirtschaft, Tübingen

Pielow, J.-C. (2002): Frankreich - Service Public, in: Hrbek, R./Nettesheim, M. (Hrsg.): Europäisches Union und mitgliedstaatliche Daseinsvorsorge, Baden-Baden, S. 155-173

Püttner, G. (2002): Das grundlegende Konzept der Daseinsvorsorge. Kommunale Daseinsvorsorge - Begriff, Geschichte, Inhalte, in: Hrbek, R./ Nettesheim, M. (Hrsg.): Europäisches Union und mitgliedstaatliche Daseinsvorsorge, Baden-Baden, S. 32-38

Scheidemann, D. (1991): Der Begriff Daseinsvorsorge, Göttingen Schneider, K. (2006): Öffentliche Daseinsvorsorge - die europäische Dimension, in: Kißler, L./Lasserre, R./Pautrat, M.-H. (Hrsg.): Öffentlicher Dienst und Personalmanagement. Zur Verwaltungsreform in Deutschland und Frankreich, Frankfurt a. M./New York, S. 27-37

Sturm, R./Müller, M. (2002): Die Reform der Daseinsvorsorge im deutsch-britischen Vergleich, in: Hrbek, R./Nettesheim, M. (Hrsg.): Europäische Union und mitgliedstaatliche Daseinsvorsorge, Baden-Baden, S. $174-198$ 